\title{
Naturally occurring Polyhydroxyalkanoate (PHA)- based scaffolds used for in vitro tissue engineering applications
}

\author{
Eleni Chatzilakou, Olga Tsave, Christos Chatzidoukas* \\ Department of Chemical Engineering/ Aristotle University of Thessaloniki \\ University Campus,54124, Thessaloniki, Greece \\ chatzilak@cheng.auth.gr, tsaveolga@auth.gr, chatzido@auth.gr
}

\section{Extended Abstract}

Metal alloys have been used extensively as medical implants, nevertheless, various data correlating metal traces with the development of immune-related adverse events, have emerged raising safety concerns. PHAs constitute a class of functional biomaterials with auspicious properties. To this end, the aim of this study was the production of three biomedically promising PHAs, namely poly-hydroxybutyrate (PHB), poly-hydroxybutyrate-co-valerate (PHBV) and polyhydroxyoctanoate (PHO), followed by the characterization of their mechanical and physical-chemical properties prior to assessing their biological profile with well-established in vitro models.

The bacterial cultures and the simultaneous intracellular biopolymer synthesis were performed in a lab-scale bioreactor, employing established fed-batch cultivation protocols [1]. The bacterial strain Pseudomonas putida and Azohydromonas lata were cultivated to acquire PHO and PHB, PHBV, respectively. Subsequently, the mechanical disruption of cell membranes and the extraction of the intercellular biopolymer were performed.

In order to tailor the mechanical properties and architecture of the scaffolds, their correlation with the dissolution time, the $\% \mathrm{w} / \mathrm{v}$ polymer/chloroform ratio, and the solvent evaporation technique was studied. Accordingly, the complete characterization of their physical-chemical, molecular, and mechanical properties, applying Scanning Electron Microscopy, Differential Scanning Calorimetry, BET Surface Analysis, Tensile-Compressive Strength testing, Gel Permeation and Gas Chromatography, was performed.

Three cell lines, namely 3T3-L1 (mouse pre-adipocytes), SaOs-2 (human bone osteosarcoma) and C2C12 (mouse myoblasts) were examined. All cell lines were seeded in appropriate culture plates coated with the PHAs, tested in terms of biological assays for cell adhesion, toxicity, proliferation, and differentiation and juxtaposed with those seeded in plane Tissue Culture Plastic. Cell adhesion was assessed through monitoring metabolically active cells, while cell viability and proliferation were determined by Sulforhodamine-B toxicity assay. Regarding differentiation, 3T3-L1 cells were differentiated into mature adipocytes according to standard differentiation protocol [2]. Successful adipogenesis was confirmed by Oil-red-O staining. C2C12 were differentiated in the presence of $2 \%$ horse serum, until mature myotubes were observed. SaOs-2 cells were induced to differentiate by adding ascorbic acid, refreshing the medium every third day for 27 consecutive days. Successful mineralization was tested by Alizarin- Red staining.

Polymer recovery techniques were implemented to obtain sufficient amounts of biopolymers with minimum implication at their molecular properties. The mechanical and physicochemical measurements indicated that the produced biomaterials are qualified for tissue engineering applications. The film formation study led to the optimization of the applied protocol, acquiring homogeneous and unfissured films, suitable for biomimetic purposes.

Concerning the examination of the biomedical suitability of the scaffolds, successful cell adhesion was achieved as monolayers for all cell lines. Regular examination of cell morphology indicated no aberration from the healthy state and no reduction in cell viability was observed. Cell differentiation was successful in all cases examined. In conclusion, the aforementioned results render PHB, PHBV and PHO as valuable candidates to meet the demanding biomedical and tissue engineering requirements.

\section{References}

[1] G. Penloglou, C. Chatzidoukas, C. Kiparissides, Biotechnology Advances, 30 329-337 (2012)

[2] O. Tsave, M.P. Yavropoulou, M. Kafantari, C.Gabriel, J.G. Yovos, A. Salifoglou, Inorg. Biochem.186 217-227 (2018) 